Copyright (c) 2006 IEEE. Reprinted from

IEEE Signal Processing Letters, 2006; 13 (8):477-480

This material is posted here with permission of the IEEE. Such permission of the IEEE does not in any way imply IEEE endorsement of any of the University of Adelaide's products or services. Internal or personal use of this material is permitted. However, permission to reprint/republish this material for advertising or promotional purposes or for creating new collective works for resale or redistribution must be obtained from the IEEE by writing to pubs-permissions@ieee.org.

By choosing to view this document, you agree to all provisions of the copyright laws protecting it. 


\title{
Complex Rational Orthogonal Wavelet and Its Application in Communications
}

\author{
Limin Yu and Langford B. White, Senior Member, IEEE
}

\begin{abstract}
This letter proposes a generalized method to construct complex wavelets under the framework of rational multiresolution analysis, $\operatorname{MRA}(M)$, where $M$ is a rational number. Theorems and examples are given for the construction of complex rational orthogonal wavelets (CROWs) whose real and imaginary parts form an exact Hilbert transform pair. Since the classical Mallat's MRA is a special case of the rational $\operatorname{MRA}(M)$ with $M=2$, the theorems hold for the construction of complex dyadic wavelets. Based on a rational MRA $(M)$ with $1<M<2$, the constructed CROWs not only achieve the benefit by capturing the phase information that the real-valued wavelets are lacking but also have the unique fine rational orthogonal property that is suited for specific application scenarios where binary orthogonality achieved by dyadic wavelets is not sufficient for the scale resolution. The CROWs' application in communications as the modulation signal pulse for PSK/QAM signaling is discussed. Specific communication scenarios that could benefit from the properties of this class of complex wavelets include the communication through a multipath/Doppler channel and new CROW-based multicarrier modulation (MCM)/orthogonal frequency division multiplexing (OFDM) systems.
\end{abstract}

Index Terms-Complex rational orthogonal wavelet (CROW), complex wavelet, Hilbert transform pair, multicarrier modulation (MCM), multipath/Doppler, orthogonal frequency division multiplexing (OFDM), rational multiresolution analysis (MRA).

\section{INTRODUCTION}

$\mathbf{C}$ OMPLEX wavelets featuring both amplitude and phase information outperform real-valued wavelets (featuring only the amplitude information) in many applications where local phase information of signals or images is needed. There are numbers of ways to construct complex wavelets with variant properties. Complex Gaussian, complex Morlet, and complex frequency B-spline wavelets are defined in the time domain with explicit expressions [1]. They are not orthogonal and compactly supported; therefore, there are no discrete wavelet transform (DWT) and finite impulse response (FIR) analysis/synthesis filters. Complex wavelets were also constructed by designing complex conjugate quadrature filters (CQFs), which lead to the associate complex wavelets [2]. Further efforts have been focused on the design of pairs of wavelet filters to achieve desired orthogonality and symmetry properties of complex wavelet transforms [3]-[5].

In this letter, we propose a generalized method to construct complex wavelets under the framework of rational multireso-

Manuscript received November 3, 2005; revised January 20, 2006. The associate editor coordinating the review of this manuscript and approving it for publication was Dr. Hakan Johansson.

The authors are with the School of Electrical and Electronic Engineering, The University of Adelaide, Adelaide SA 5005, Australia (e-mail: liminyu@eleceng.adelaide.edu.au; lang.white@adelaide.edu.au).

Digital Object Identifier 10.1109/LSP.2006.874393 lution analysis, $\operatorname{MRA}(M)$, where $M$ is a rational number. It has been pointed out that we can always take the Hilbert transform of one wavelet to form another wavelet [4]. We give a theoretical verification of this argument and prove that complex wavelets with nice properties can be derived based on the two related wavelets. Theorems and examples are given for the construction of complex rational orthogonal wavelets (CROWs) with the rational orthogonal property and whose real and imaginary parts form an exact Hilbert transform pair. Since the classical Mallat's MRA is a special case of the rational MRA $(M)$ with $M=2$, these theorems also hold for the construction of complex dyadic wavelets. Based on a rational $\operatorname{MRA}(M)$ with $1<M<2$, a new class of CROWs is constructed. Besides the benefit it achieves by capturing the phase information that the real-valued wavelets are lacking, it has the unique rational orthogonal property that is suited for specific application scenarios where binary orthogonality achieved by dyadic wavelets is not sufficient for the scale resolution. The CROWs' application in communications for orthogonal frequency division multiplexing (OFDM) transmission and the communication through a multipath/Doppler channel is discussed.

This letter is organized as follows. In Section II, the theorems for the construction of the CROWs are presented. Examples of the constructed complex wavelets are given in Section III. The CROWs' application in communications is discussed in Section IV. Section V presents the conclusions of this letter.

\section{CONSTRUCTION OF COMPLEX RATIONAL ORTHOGONAL WAVELETS}

\section{A. Rational MRA and Real Rational Orthogonal Wavelets}

Real rational orthogonal wavelets were derived under the framework of a rational MRA proposed by Auscher in [8]. The author addressed the problem that given any real number $M>1$, does there exist a finite set, $\psi_{1}, \psi_{2}, \ldots, \psi_{\ell}$, of functions in $L^{2}(\mathbb{R})$ such that the family $M^{j / 2} \psi_{n}\left(M^{j} x-k\right), j, k \in \mathbb{Z}, 1 \leq n \leq \ell$, is an orthonormal basis for $L^{2}(\mathbb{R})$ ? A positive answer was given. An MRA with rational dilation factor $M$ was defined by the following.

Definition 1: An orthonormal MRA for $L^{2}(\mathbb{R})$ with dilation factor $M$ [denoted by $\operatorname{MRA}(M)$ ] consists of a collection $V_{j}$, $j \in \mathbb{Z}$, of subspaces such that

1) $V_{j} \subset V_{j+1}, \forall j \in \mathbb{Z}$

2) $\bigcap_{j \in \mathbb{Z}} V_{j}=\{0\}$ and $\bigcup_{j \in \mathbb{Z}} V_{j}$ is dense in $L^{2}(\mathbb{R})$;

3) $f(x) \in V_{j}$ if and only if $f(M x) \in V_{j+1}$;

4) there exists a function $\phi(x) \in V_{0}$ such that $\{\phi(x-k), k \in$ $\mathbb{Z}$ \} forms an orthonormal basis for $V_{0}$.

Let $W_{j}$ be the orthogonal complement of $V_{j}$ in $V_{j+1}$. The following theorem for the wavelet basis related to an $\operatorname{MRA}(M)$ is borrowed from [8]. 
Theorem 1: With Definition 1 and assuming $M=p / q, p$ and $q$ being relatively prime integers with $p>q>1$, there exists a set of $p-q$ wavelet functions $\psi_{1}, \psi_{2}, \cdots, \psi_{p-q}$ in $W_{0}$ such that the collection of functions $(p / q)^{j / 2} \psi_{n}\left((p / q)^{j} x-k q\right), k, j \in$ $\mathbb{Z}, 1 \leq n \leq p-q$ forms an orthonormal wavelet basis for $L^{2}(\mathbb{R})$.

It was further pointed out that the number of wavelets in $W_{0}$ does not depend on the choice of the $\psi_{i}$ 's, and the choice of the $\psi_{i}$ 's is not unique. We will restrict our extension to the complex wavelets for certain cases where $M=1+(1 / q)$ and therefore a single $\psi$ can be chosen with desired regularity and decay property. Our method of construction, however, applied to general $M$. In the sequel, we will denote scaling factors of this form by a.

\section{B. Theorems for the Construction of Complex Rational Orthogonal Wavelets}

Assume there is an $\operatorname{MRA}(a)$, where $a=1+(1 / q)$ and $\phi(t)$ and $\psi(t)$ are the related scaling function and wavelet function, respectively. We claim the following.

1) The collection of $\hat{V}_{j}, j \in \mathbb{Z}$, where $\hat{V}_{j}=\left\{\sum_{k} b_{k} \hat{\phi}\left(a^{j}\right.\right.$. $\left.k),\left\{b_{k}\right\} \in \ell_{2}\right\}$, forms another $\operatorname{MRA}(a)$ where $\hat{\phi}(t)$ is the Hilbert transform of $\phi(t)$.

2) Letting $\hat{W}_{j}$ be the orthogonal complement of $\hat{V}_{j}$ in $\hat{V}_{j+1}$, there exists a wavelet function $\hat{\psi}(t)$ in $\hat{W}_{0}$ such that the collection of functions $\left\{\hat{\psi}_{k, n}(t):=a^{-k / 2} \hat{\psi}\left(a^{k} t-n q\right), k, n \in\right.$ $\mathbb{Z}\}$ forms an orthonormal wavelet basis for $L^{2}(\mathbb{R})$, where $\hat{\psi}(t)$ is the Hilbert transform of $\psi(t)$.

The above claims can be further summarized as the following theorem.

Theorem 2: If there are a scaling function $\phi(t)$ and a wavelet basis function $\psi(t)$ associated with an $\operatorname{MRA}(a)$ defined in Definition 1 and $a=1+(1 / q), q \in \mathbb{Z}^{+}$, the Hilbert transform of $\psi(t), \hat{\psi}(t)$ is also a wavelet basis function associated with another $\operatorname{MRA}(a)$ with the orthogonality, $\int \hat{\psi}_{k n}(t) \hat{\psi}_{\ell m}(t) d t=$ $\delta(k-\ell) \delta(m-n), k, \ell, m, n \in \mathbb{Z}$, such that the collection of functions $\left\{\hat{\psi}_{k, n}(t):=a^{-k / 2} \hat{\psi}\left(a^{k} t-n q\right), k, n \in \mathbb{Z}\right\}$ is an orthonormal basis of $L^{2}(\mathbb{R})$. There exists the corresponding scaling function $\hat{\phi}(t)$, which is the Hilbert transform of $\phi(t)$.

Proof: The proof of 4) in Definition 1 for claim 1) is independent of the choice of $a$ and reduces to the following identity: $\sum_{k \in \mathbb{Z}}|\hat{\Phi}(\omega+2 k \pi)|^{2}=1, \omega \in \mathbb{R}$. Since we have $|\hat{\Phi}(\omega)|=|\Phi(\omega)|$ and $\sum_{k \in \mathbb{Z}}|\Phi(\omega+2 k \pi)|^{2}=1$, the verification is immediate. The proof of 1) in Definition 1 is as follows: Since $V_{j} \subset V_{j+1}$, we have $\phi_{j, n}(t)=\sum_{k \in \mathbb{Z}} b_{n, k} \phi_{j+1, k}(t)$, $n=0,1, \cdots, q-1$ [9], which is equivalent to $\Phi\left(a^{-j} \omega\right)=$ $a^{-1 / 2} \sum_{k \in \mathbb{Z}} b_{n, k} e^{-i k a^{-(j+1)} \omega} e^{-i n a^{-j} \omega} \cdot \Phi\left(a^{-(j+1)} \omega\right)$

for some $\left\{b_{n, k}\right\} \in \ell_{2}$. From the definition of Hilbert transform, we have $\hat{\Phi}(\omega)=-i \operatorname{sgn}(\omega) \Phi(\omega)$, where the Sgn function $\operatorname{sgn}($.$) returns the sign of number \omega$. Therefore, we have $\hat{\Phi}\left(a^{-j} \omega\right)=a^{-1 / 2} \sum_{k \in \mathbb{Z}} b_{n, k} e^{-i k a^{-(j+1)} \omega}$. $e^{-i n a^{-j} \omega}(-i) \operatorname{sgn}(\omega) \Phi\left(a^{-(j+1)} \omega\right)$

$a^{-1 / 2} \sum_{k \in \mathbb{Z}} b_{n, k} e^{-i k a^{-(j+1)} \omega} e^{-i n a^{-j} \omega} \hat{\Phi}\left(a^{-(j+1)} \omega\right)$. This proves $\hat{V}_{j} \subset \hat{V}_{j+1}$. The verification of 2) and 3) is immediate with the proof of 1) and 4).

The proof of claim 2) consists of three points: a) the orthonormality of $\hat{\psi}(t)$ that the set $\{\hat{\psi}(t-k q), k \in \mathbb{Z}\}$ forms an orthonormal basis for $\hat{W}_{0}$; b) $\hat{W}_{j} \subset \hat{V}_{j+1}, j \in \mathbb{Z}$; and c) $\hat{W}_{j} \perp \hat{V}_{j}$, $j \in \mathbb{Z}$. First, since we have the orthonormality of $\psi(t)$ that
$\sum_{k}|\Psi(\omega+2 k q \pi)|^{2}=1$, and there is $|\hat{\Psi}(\omega)|=|\Psi(\omega)|$, it follows that $\sum_{k}|\hat{\Psi}(\omega+2 k q \pi)|^{2}=1$, which proves the first point. For the proof of the second point, it is similar to the proof of $\hat{V}_{j} \subset \hat{V}_{j+1}$. Since we have $W_{j} \subset V_{j+1}$, which gives us the relation $\Psi\left(a^{-j} \omega\right)=\sum_{k \in \mathbb{Z}} d_{k} e^{-i k w} \Phi\left(a^{-(j+1)} \omega\right)$, the Hilbert transform of $\Psi\left(a^{-j} \omega\right)$ can be expressed as $\hat{\Psi}\left(a^{-j} \omega\right)=$ $\sum_{k \in \mathbb{Z}} d_{k} e^{-i k w} \hat{\Phi}\left(a^{-(j+1)} \omega\right)$. This proves $\hat{W}_{j} \subset \hat{V}_{j+1}$. For the third point, by the Parseval formula, the orthogonality of $\hat{W}_{j}$ to $\hat{V}_{j}$ is equivalent to $\hat{I}_{\ell}=\int_{\mathbb{R}} \hat{\Psi}(\omega) \hat{\Phi}^{*}(\omega) e^{-i \ell \omega} d \omega=0$. It can be derived directly from the condition that $W_{j} \perp V_{j}$, which has the relation $I_{\ell}=\int_{\mathbb{R}} \Psi(\omega) \Phi^{*}(\omega) e^{-i \ell \omega} d \omega=0$, and there is $\hat{I}_{\ell}=\int_{\mathbb{R}}(-i) \operatorname{sgn}(\omega) \Psi(\omega) i \operatorname{sgn}(\omega) \Phi^{*}(\omega) e^{-i \ell \omega} d \omega=I_{\ell}=0$.

Define complex functions $\phi_{+}(t)=\phi(t)+i \hat{\phi}(t)$, and $\psi_{+}(t)=$ $\psi(t)+i \hat{\psi}(t)$. Applying the condition to $\psi(t)$ that

$$
<\psi_{k, n}(t), \hat{\psi}_{l, m}(t)>\backslash=0, k, n, l, m \in \mathbb{Z}
$$

we have the following theorem.

Theorem 3: If there are a scaling function $\phi(t)$ and a wavelet basis function $\psi(t)$ associated with an $\operatorname{MRA}(a), a=1+\frac{1}{q}, q \in$ $\mathbb{Z}^{+}$, the complex function $\psi_{+}(t)$ satisfying the condition (1), is a complex wavelet basis function associated with a complex $\operatorname{MRA}(a)$ for a complex functional space $L^{2}(\mathbb{R})$ with the orthogonality $\int \psi_{+, k n}(t) \psi_{+, l m}^{*}(t) d t=\delta(k-l) \delta(m-n), k, l, m, n \in$ $\mathbb{Z}$, such that the collection of complex functions $\left\{\psi_{+, k n}(t):=\right.$ $\left.\frac{\sqrt{2}}{2} a^{k / 2} \psi_{+}\left(a^{k} t-n q\right), k, n \in \mathbb{Z}\right\}$ is an orthonormal basis of the complex valued $L^{2}(\mathbb{R})$. The complex function $\phi_{+}(t)$ is the corresponding complex scaling function.

The proof of Theorem 3 is verified immediately based on the two real $\operatorname{MRA}(a)$ 's and the orthonormality between the two sets of real wavelet subspaces. Notice that the condition (1) imposes a constraint on the spectrum of $\psi(t)$ that $\operatorname{Im}\left[\int_{0}^{\infty} \Psi\left(a^{k} \omega\right) \Psi^{*}(\omega) e^{-i \omega q\left(a^{k} n-m\right)} d \omega\right]=0, k, n, m \in \mathbb{Z}$, where $\operatorname{Im}[\cdot]$ obtains the imaginary part of a complex signal. In the next section, we give examples that satisfy this condition approximately, while finding appropriate wavelets, e.g., Littlewood Paley wavelet, where exact orthogonality is an open question.

It is worth noting that the Hilbert transform cancels the mean value of the signal; therefore, the spectrum of $\hat{\phi}(t)$ has the discontinuity at zero frequency, $\hat{\Phi}(0)=0$. This discontinuity can be neglected if we cope with signals of zero mean or having the mean value [the direct current (DC) component] removed before processing, which is the case for most of the signal processing problems. For the spectrum equality $|\hat{\Phi}(\omega)|=|\Phi(\omega)|$ and $\left\{\hat{\phi}_{j}(t-k), k, j \in \mathbb{Z}\right\}$ being the Riez basis of $\hat{V}_{j}$ and $\bigcup_{j \in \mathbb{Z}} \hat{V}_{j}=L^{2}(\mathbb{R})$ to be exact, we may define $\hat{\Phi}(0)$ additionally by $|\hat{\Phi}(0)|=\left|\hat{\Phi}\left(0^{+}\right)\right|$or $\left|\hat{\Phi}\left(0^{-}\right)\right|$in order to relax the constraint on the spaces $\left\{\hat{V}_{j}, j \in \mathbb{Z}\right\}$ spanned by functions with zero mean. The wavelet subspaces $\hat{W}_{j}$ are not affected by this property of Hilbert transform because of the bandpass property of $\psi(t)$.

Notice that with the method proposed in this letter, the two $\operatorname{MRA}($ a)s related to $\{\phi(t), \psi(t)\}$ and $\{\hat{\phi}(t), \hat{\psi}(t)\}$ have the same two-scale relations between the neighboring subspaces. For the dyadic case $(a=2)$, Selecnick [4] proposed another way that leads to a pair of wavelets forming the Hilbert transform pair by designing two sets of lowpass and highpass filters that converge to the corresponding scaling functions 
and wavelets. One of the scaling functions is the time-shifted version of the other by $1 / 2$. It is different from the Hilbert transform relation of the scaling functions in this letter because the two MRA(2)s in [4] are based on two different sets of two-scale relations. This observation infers that for the same Hilbert transform pair of wavelets, the relation between the two scaling functions is not unique, depending on the two-scale relations between the neighboring subspaces of the two $\operatorname{MRA}(a)$ 's.

\section{EXAMPLES OF COMPLEX RATIONAL ORTHOGONAL WAVELETS}

In this section, we construct the CROWs based on the definition of real rational orthogonal wavelets (RROWs) with dilation factor $a=1+(1 / q)$ given in [6]. The corresponding rational wavelet basis function $\psi(t)$ is defined in the frequency domain by

$$
\Psi(\omega)=\left\{\begin{array}{cc}
(2 \pi)^{-\frac{1}{2}} e^{j \frac{\omega}{2}} & \\
\times \sin \left(\frac{\pi}{2} \beta\left(\frac{q}{\omega_{1}}|\omega|-q\right)\right), & \omega_{1} \leq|\omega| \leq \omega_{2} \\
(2 \pi)^{-\frac{1}{2}} e^{j \frac{w}{2}} & \\
\times \cos \left(\frac{\pi}{2} \beta\left(\frac{q}{\omega_{2}}|\omega|-q\right)\right), & \omega_{2} \leq|\omega| \leq \omega_{3} \\
0, & |\omega| \notin\left[\omega_{1}, \omega_{3}\right]
\end{array}\right.
$$

and the rational scaling function $\phi(t)$ is defined by

$$
\Phi(\omega)= \begin{cases}(2 \pi)^{-\frac{1}{2}}, & |\omega| \leq \omega_{1} \\ (2 \pi)^{-\frac{1}{2}} \cos \left(\frac{\pi}{2} \beta\left(\frac{q}{\omega_{1}}|\omega|-q\right)\right), & \omega_{1} \leq|\omega| \leq \omega_{2} \\ 0, & |\omega|>\omega_{2}\end{cases}
$$

where

$$
\omega_{1}=\left(q-\frac{q}{2 q+1}\right) \pi, \omega_{2}=a \omega_{1}, \omega_{3}=a \omega_{2}=a^{2} \omega_{1}
$$

and $\beta(t)$ is the construction function, which is not unique. One construction function that leads to desirable decay property of the wavelet is given by $\beta(t)=t^{4}\left(35-84 t+70 t^{2}-20 t^{3}\right)$.

Based on the definition in (2), we define the complex wavelet function as

$$
\psi_{+}(t)=\psi(t)+j \hat{\psi}(t)
$$

where $\psi(t)$ has the frequency spectrum defined in (2), and $\hat{\psi}(t)$ is also defined in the frequency domain by

$$
\hat{\Psi}(\omega)=-j \operatorname{sqn}(\omega) \Psi(\omega)
$$

so that $\hat{\psi}(t)$ is the Hilbert transform of $\psi(t)$. The real part and imaginary part of the complex wavelet form a Hilbert transform pair. $\psi_{+}(t)$ is actually the analytic form of the RROW $\psi(t)$.

We see that three wavelets are obtained based on the definitions in (2), (5), and (6), respectively. The first two, $\psi(t)$ and (a)

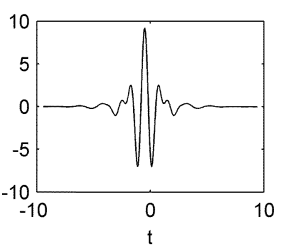

(d)

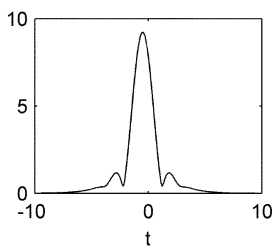

(b)

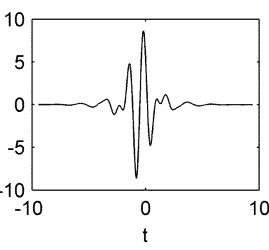

(e)

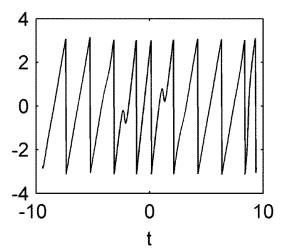

(c)

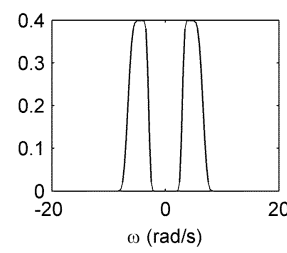

(f)

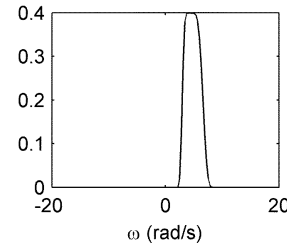

Fig. 1. Complex Meyer wavelet with dilation factor of 2. (a) Real part of $\psi_{+}^{(2)}(t)$. (b) Imaginary part of $\psi_{+}\left({ }^{(2)}(t)\right.$. (c) Spectrum amplitude of both real and imaginary parts. (d) Modulus of $\psi_{+}^{(2)}(t)$. (e) Angle of $\psi_{+}^{(2)}(t)$. (f) Spectrum amplitude of $\psi_{+}^{(2)}(t)$. (a)

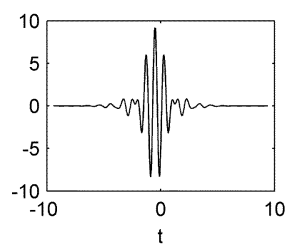

(d)

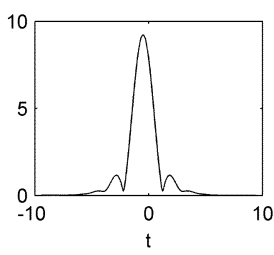

(b)

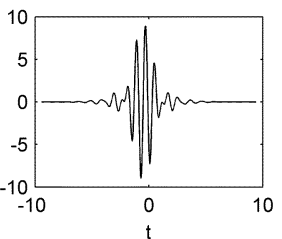

(e)

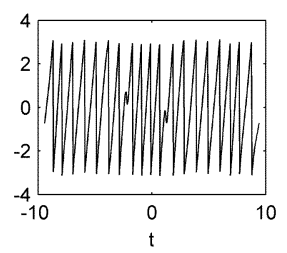

(c)

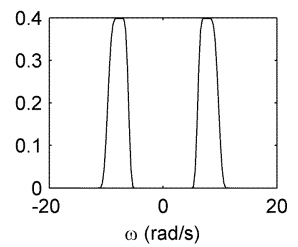

(f)

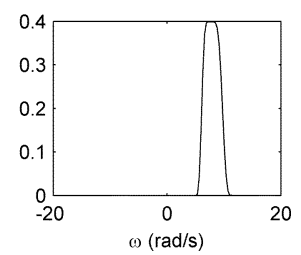

Fig. 2. Complex rational orthogonal wavelet with dilation factor of 3/2. (a)-(f) Corresponding figures for $\psi_{+}^{(3 / 2)}(t)$, as specified in Fig. 1 .

$\hat{\psi}(t)$, are real wavelets, and the third one, $\psi_{+}(t)$, is a complex wavelet. $\psi(t)$ and $\psi_{+}(t)$ are symmetric, and $\hat{\psi}(t)$ is antisymmetric. The frequency spectrum of the complex wavelet, $\Psi_{+}(\omega)$, is supported on $\omega \geq 0$. Two examples of designed complex wavelets are shown in Figs. 1 and 2. The orthogonality can be examined by evaluating $<\psi_{k, n}(t), \hat{\psi}_{l, m}(t)>\backslash=$ $0, k, n, l, m \in \mathbb{Z}$ numerically. The result shows that the complex Meyer wavelet with $q=1$ is not orthogonal with relatively large inner products at $k=l, m-n= \pm 1$, while the inner products for $\psi_{+}^{(1+1 / q)}, q>1$ are very close to zero; therefore, the orthogonal condition holds with high fidelity.

\section{APPLICATION IN COMMUNICATIONS}

\section{A. CROW-Based PSK/QAM Signaling and OFDM Transmission}

One application of the proposed CROW is to employ it as the modulation signal pulse, especially in an MCM or orthogonal multiplexing system. We give the baseband characterization of the CROW-based PSK/QAM signaling without involving the sinusoidal carrier modulation. For simplicity, a single wavelet 
signaling is considered in the derivation. For QAM modulation, the transmitted signal waveforms are represented as

$$
\begin{aligned}
s_{m}(t)= & \operatorname{Re}\left[\left(A_{m c}+j A_{m s}\right) \psi_{+}(t)\right] \\
= & A_{m c} \psi(t)-A_{m s} \hat{\psi}(t) \\
= & V_{m} \cos \theta_{m} \psi(t)-V_{m} \sin \theta_{m} \hat{\psi}(t) \\
& m=1, \cdots, M
\end{aligned}
$$

where $V_{m}=\sqrt{A_{m c}^{2}+A_{m s}^{2}}$ and $\theta_{m}=\tan ^{-1}\left(A_{m s} / A_{m c}\right)$. PSK modulation can be regarded as a special case of QAM with $V_{m}=1$. In both cases, the transmitted signal is the real part of the signal with complex wavelet pulse shaping. The symbol sequence is mapped to the complex amplitude of the pulse sequence. The transmitted signal is a linear combination of two orthogonal wavelets $\psi(t)$ and $\hat{\psi}(t)$ with $\langle\psi(t), \hat{\psi}(t)\rangle=\int \psi(t) \hat{\psi}(t) d t=0$. One specific scenario that benefits from the proposed CROWs is orthogonally multiplexed communication or wavelet-based OFDM transmission. Following the derivation in , the BW efficiency of the CROW-based OFDM signal is derived as

$$
\rho_{\mathrm{CROW}}=\frac{B}{1+\beta} \frac{2 \pi}{a^{2} \omega_{1}}\left(1+\sum_{i=1}^{J} a^{l_{i}}\right)=\frac{B}{1+\beta}\left(1+a^{-1}\right)
$$

where $B$ is the number of bits per symbol. $\beta$ is the percent excess bandwidth required beyond the Nyquist signaling bandwidth. The scale $l_{i}$ 's come from the rational overlapped partition of the interval $[0,1)$ with dilation factor of $a$, and there is $\sum_{i=1}^{J} a^{l_{i}}=$ $q$. The factor $2 \pi / a^{2} \omega_{1}$ is introduced for normalization purposes because the spectrum of the wavelet basis is localized between $\omega_{1}$ and $a^{2} \omega_{1}$, as shown in (2) and (4). For comparison, as derived in , the BW efficiency of the dyadic wavelet- and wavelet packet-based OFDM signals with dyadic partition of the interval $[0,1)$ and the spectrum of the wavelet basis function localized between $\pi$ and $2 \pi$ are given as $\rho_{\mathrm{DWM}}=\rho_{\mathrm{WPM}}=B /(1+\beta)$. The BW efficiency $\rho_{\text {CROW }}$ has a gain of $\left(1+a^{-1}\right)$ over $\rho_{\text {DWM }}$ and $\rho_{\mathrm{WPM}}$.

\section{B. CROW-Based Communication for Multipath/Doppler Channels}

Besides the CROW-based OFDM communication, another application scenario that benefits from the proposed wavelet is the communication through a multipath/Doppler channel. By utilizing the rational orthogonal wavelet as the signaling pulse, a single-carrier signal transmission through a frequency-selective multipath/Doppler channel is equivalent to an $L$ th-order diversity system with independent modulation through $L$ flat-fading subchannels, where $L$ is the number of eigenpaths. The multiscale nature [6] of the channel is resolved, and ISI/ICI cancellation is achieved due to the rational orthogonality of the signaling pulse. The optimum receiver design and system characterization for PAM signaling with real rational orthogonal wavelet were presented in [7]. The CROW proposed in this letter provides a solution addressing the PSK/QAM signaling through the multipath/Doppler channel with the CROW as the complex signaling pulse. The extension from [7] is straightforward.

\section{CONCLUSION}

In this letter, a generalized method to construct complex wavelets under the framework of rational MRA is presented. The constructed complex rational orthogonal wavelet has many nice properties, including

- explicit expression in the frequency domain;

- rational orthogonality;

- compact support in the frequency domain;

- effective support in the time domain by $[-8,8]$;

- symmetry—-symmetry for the real part and anti-symmetry for the imaginary part;

- quadrature between real and imaginary parts.

It is a promising signal processing tool for many application fields in image processing and communications. Since the wavelet has infinite support in the time domain, there is no FIR solution of the analysis/synthesis filters for the tree-structured fast wavelet transform (FWT). An FWT implementation is available, however, in the frequency domain, as shown in [9], or by FIR-based approximation as the derivation of discrete Meyer (dMeyer) wavelet from Meyer wavelet. For a parallel filter bank structure, more efficient implementation is available via FFT-based algorithms and can benefit from advanced DSP techniques on parallel calculations. Specific communication scenarios that could benefit from the properties of this class of complex wavelets include the communication through a multipath/Doppler channel and the new CROW-based MCM/OFDM systems.

\section{REFERENCES}

[1] A. Teolis, Computational Signal Processing With Wavelets. Cambridge, MA: Birkhaüser, 1998.

[2] W. Lawton, "Applications of complex valued wavelet transforms to subband decomposition," IEEE Trans. Signal Process., vol. 41, no. 12, pp. 3566-3568, Dec. 1993.

[3] N. Kingsbury, "Complex wavelets for shift invariant analysis and filtering of signals," J. Appl. Comput. Harm. Anal., vol. 10, no. 3, pp. 234-253, 2001.

[4] I. W. Selesnick, "The design of approximate Hilbert transform pairs of wavelet bases," IEEE Trans. Signal Process., vol. 50, no. 5, pp. 1144-1152, May 2002.

[5] D. B. H. Tay and M. Palaniswami, "Design of approximate Hilbert transform pair of wavelets with exact symmetry," in Proc. ICASSP, 2004, vol. 2, pp. 921-924.

[6] L. Yu and L. B. White, "Broadband Doppler compensation for rational wavelet-based UWA communication systems," in Proc. Asia-Pacific Conf. Communications (APCC), 2005, pp. 605-609.

[7] _ "Optimum receiver design for broadband Doppler compensation in multipath/doppler channels with rational orthogonal wavelet signalling part 1: Theory and properties," IEEE Trans. Signal Process., submitted for publication.

[8] P. Auscher, "Wavelet bases for $\mathrm{L}^{2}(\mathrm{R})$ with rational dilation factor," in Wavelets and Their Applications. Boston, MA: Jones \& Barlett, 1992, pp. 439-451.

[9] A. Baussard, F. Nicolier, and F. Trucheter, "Rational multiresolution analysis and fast wavelet transform: Application to wavelet shrinkage denoising," Signal Process., vol. 84, pp. 1735-1747, 2004. 